Methods We performed a cohort study among HCWs of a large University hospital in Milan, Lombardy, Italy by merging routinely collected data on demographics, COVID-19 vaccination, and polymerase chain-reaction (PCR) tests performed on nasopharyngeal swabs. Follow-up started on December 27, 2020 (start of vaccination campaign). We included HCWs never PCR-positive before the start date and with at least a PCR test afterwards. Vaccination was treated as a timedependent variable by calculating person-years (PY) at risk before and after vaccine doses. Subjects contributed PY until first positive PCR test (cases) or last test for never positive HCWs (to avoid immortal time bias). We calculated infection rates (cases per $1000 \mathrm{PY}$ ), rate ratios (RR, with a Poisson regression model adjusted for gender, age, occupation and 30day periods), vaccine effectiveness $(\mathrm{VE}=(1-\mathrm{RR}) \mathrm{x} 100)$ and $95 \%$ confidence intervals (CI) taking never vaccinated HCWs as reference.

Results As of May 10, there were 3,152 vaccinated (97\% with BNT162b2, 140 with one dose, 2,679 with two doses) and 333 non-vaccinated. We counted 29 infected cases (rate 385) among non-vaccinated, 6 (rate 65) from day 14 after the first dose (VE 79\%, CI 49-92\%), and 24 (rate 65) from day 7 after the second dose (VE 89\%, CI 80-94\%). Most cases after vaccination were asymptomatic or mildly symptomatic.

Conclusion In these preliminary analysis we found high effectiveness of COVID-19 vaccine in HCWs in our hospital. Further work is needed to assess long-term effectiveness and to better plan future preventive strategies among this high-risk occupational group.

\section{P-196 PREVALENCE OF NIGHT/SHIFT WORK AND ITS ASSOCIATION WITH EXITING THE WORKFORCE AMONGST OLDER UK WORKERS FROM THE HEAF STUDY}

${ }^{1}$ Gregorio Bevilacqua, Stefania D'Angelo, Georgia Ntani, Martin Stevens, Cathy Linaker, Karen Walker-Bone. 'University of Southampton, United Kingdom

\subsection{6/OEM-2021-EPI.229}

Introduction Night/shift work is increasing but there are few data about the prevalence amongst older workers. Night/shift work has been associated with a number of adverse physical and mental health outcomes, including cardiovascular disease, cancer, anxiety, and depression. With governments encouraging people to work to older ages, it is important to know how feasible night/shift work is for older workers and whether it is associated with prematurely exiting the workforce.

Objectives Amongst current older workers (aged 50-64 years), to explore the prevalence of night/shift working and evaluate its associations with early exit from the workforce over 4 years of follow-up.

Methods Data from the Health and Employment After Fifty (HEAF) cohort were used to describe the demographic, job and health characteristics of men and women undertaking night/shift work. Longitudinal data from annual follow-ups were used to examine the number and nature of exits annually thereafter.

Results Amongst the 5409 working at baseline, 32\% reported night/shift work but the sectors differed by sex. Night/shift workers were more likely to be: current smokers; doing physically-demanding work; struggling to cope at work physically and mentally; dissatisfied with their hours; depressed; sleeping poorly; and/or rating their health poorly. Men (OR 1.4, 95\%CI 1.1-1.8) and women (OR 1.3, 95\%CI 1.0-1.6) working nights/shifts were slightly more likely to exit the workforce over 4 years. A greater proportion of those exiting the workforce who were night/shift workers attributed their exit to ill-health compared with those working conventional hours.

Conclusion In our study, almost one in three workers reported night/shift work. We found some evidence of adverse impact on health, sleep and wellbeing and higher rates of job exit in shift/night workers. More research is needed, but night/shift work may be challenging to sustain for older workers and could be detrimental to health.

\section{P-199 LONG TERM EXPOSURE TO AIR POLLUTION AND COVID-19 INCIDENCE IN THE CITY OF VARESE, NORTHERN ITALY: A COMPLETE-YEAR, INDIVIDUAL- LEVEL ANALYSIS}

${ }^{1}$ Giovanni Veronesi, Sara De Matteis, Giuseppe Calori, Nicola Pepe, Marco Ferrario. 'University of Insubria, Italy

\subsection{6/OEM-2021-EPI.230}

Introduction Studies on air pollution and COVID-19 are limited to the first pandemic wave (April/June 2020) and by their ecological design.

Objectives To investigate the association between airborne pollutants and SARS-CoV-2 incidence up to March 2021 in the Varese city (Lombardy region), with individual-level data on exposures, disease and confounders.

Methods Varese citizens aged 18+ years as of Dec31st,2019 were linked by residential address to 2018 average annual exposure to outdoor concentrations of PM2.5, PM10, NO2, $\mathrm{NO}$ and $\mathrm{O} 3$ modelled using FARM chemical-transport model (linkage coverage: 97.4\%). Citizens were linked to Regional datasets for COVID-19 case ascertainment (positive nasopharyngeal swab specimens) and to define age, sex, residential care home living, population density and comorbidities. We estimated rate ratios and additional number of COVID-19 cases for $1 \mu \mathrm{g} / \mathrm{m} 3$ increase in air pollutants, from single- and bi-pollutant Poisson regression models.

Results Among the 62.848 residents, we observed 4408 COVID-19 cases. Yearly average PM2.5 exposure was 12.5 $\mu \mathrm{g} / \mathrm{m} 3$. Cumulative incidence curves suggest an increased risk for PM2.5>13.5 $\mu \mathrm{g} / \mathrm{m} 3$ in correspondence of downtrend periods in the pandemic curve. Age, residential care home living, history of stroke, medications for diabetes, hypertension and obstructive airway disease were independently associated with COVID-19 rate. In single-pollutant multivariate model, $1 \mu \mathrm{g} /$ $\mathrm{m} 3$ increase in PM2.5 was associated with 5.1\% increase in COVID-19 rate (95\%CI: 2.7\%-7.5\%), corresponding to 294 additional cases per 100.000 person-years. These figures were confirmed in bi-pollutant models and after excluding subjects in residential care homes. Similar findings were observed for PM10, NO2 and NO. O3 was associated with a $2 \%$ decrease in disease rate, the association being reversed in bi-pollutant models.

Conclusions In our study, long term exposure to low-levels of air pollutants, especially PM2.5, positively affected COVID-19 incidence. Causality warrants confirmation in future studies; 